\title{
Real Estate trifft auf Blockchain: Chancen und Herausforderungen der Tokenisierung von illiquiden Vermögenswerten
}

\author{
Marina Markheim • Aleksander Berentsen
}

Eingegangen: 30. März 2020 / Überarbeitet: 3. Dezember 2020 / Angenommen: 22. Dezember 2020 /

Online publiziert: 12. Januar 2021

(C) Der/die Autor(en) 2021

Zusammenfassung Der vorliegende Artikel analysiert die Chancen und Herausforderungen der Tokenisierung von Immobilien. Zunächst werden die theoretischen Grundlagen (Blockchain und Smart Contracts) sowie die Vor- und Nachteile der Tokenisierung diskutiert. Die Umsetzung der Tokenisierung einer Immobilie wird anschließend anhand eines Praxisbeispiels veranschaulicht. Die Vorteile einer Tokenisierung liegen in dem sofortigen weltweiten Handel der Token rund um die Uhr, dem Eigentumsübertrag der Token in ,real time“, der Standardisierung bei gleichzeitiger Flexibilität, der Transparenz und Sicherheit im Handel der Token. Zudem ist die technische Umsetzung der Tokenisierung einer Immobilie sehr einfach. Regulatorische Unsicherheiten beschränken jedoch zurzeit noch eine breite Anwendung der Technologie.

Schlüsselwörter Blockchain Technologie - Smart Contracts · Tokenisierung der Immobilien · Regulierung der Token

M. Markheim $(\bowtie) \cdot$ A. Berentsen

Center for Innovative Finance, Universität Basel, Basel, Schweiz

E-Mail: marina.markheim@unibas.ch

A. Berentsen

E-Mail: aleksander.berentsen@unibas.ch 


\title{
Real estate meets blockchain: Opportunities and challenges of tokenization of illiquid assets
}

\begin{abstract}
This article analyses the opportunities and challenges of tokenization of real estate. The tokenization of illiquid assets serves to raise capital and manage liquidity. We discuss the theoretical foundations (blockchain and smart contracts) and discuss the advantages and disadvantages compared to a classical capital procurement. The implementation is discussed by means of a practical example. The theoretical advantages of tokenization are liquidity (immediate worldwide trading 24/7, transfer of ownership in "real time"), standardization with simultaneous flexibility, transparency and security. The most important findings can be summarized as follows: The technical creation of a real estate token is very simple, but the many theoretical advantages over traditional forms of financing are not yet being realized in practice. The reasons for this are the existing regulatory uncertainties as well as existing regulations, which make most of the theoretical advantages null and void.
\end{abstract}

Keywords Blockchain technology - Smart contracts · Tokenizing real estate · Token regulation

\section{Einleitung}

Illiquide Vermögenswerte lassen sich kurzfristig nur schwer zu ihrem tatsächlichen Wert verkaufen. Bei illiquiden Vermögenswerten handelt es sich beispielsweise um Immobilien, Kunstgegenstände und Kraftfahrzeuge. Besonders der Immobilienmarkt ist dafür bekannt, dass der Handel durch lange Transaktionszeiträume sowie hohen Transaktionskosten gekennzeichnet ist.

Die Kosten der Illiquidität werden zum Beispiel in Damodaran (2005), Cheng et al. (2013), Anson et al. (2011) und Ang (2014) untersucht. Damodaran (2005) findet einen Liquiditätsabschlag zwischen 20 und 30\%. Zu diesen Kosten zählt unter anderem die Vermittlerprovision, die beim Verkauf einer Liegenschaft im Durchschnitt zwischen fünf und sechs Prozent des Immobilienwertes beträgt. Bei Kunstoder Sammlerstücken liegen die Provisionen zwischen 15 und 20\% des Gemäldewertes.

Der Zweck der Tokenisierung einer Immobilie ist es dem Eigentümer zu ermöglichen, sich jederzeit schnell und unkompliziert liquide Mittel durch die Ausgabe von Token zu beschaffen. Aus der Sicht der Token-Käufer ist der Vorteil der Tokenisierung, dass die Token unmittelbar weltweit auf einer öffentlich zugänglichen Plattform in sehr kleiner Stückelung rund um die Uhr handelbar sind.

Ein weiterer Vorteil der Tokenisierung ist die sich abzeichnende Standardisierung. Diese Technologie wird es in der Zukunft ermöglichen, weltweit Anspruch auf den Cashflow einer Immobilie mittels Token zu erwerben, die mit dem gleichen Standard geschaffen werden. Die Standardisierung hat den Vorteil, dass ein TokenKäufer nur eine Softwareanwendung benötigt, die den unkomplizierten Empfang und Versand von Token erlaubt. Darüber hinaus wird die Standardisierung die Suche 
nach tokenisierten Immobilien vereinfachen und diese nach vergleichbaren Kriterien sortieren.

Eine breite Anwendung der Tokenisierung wird Preiseffekte haben. Der Grund dafür ist, dass diese Technologie die Erschließung neuer Märkte ermöglicht. Zudem erlaubt die Tokenisierung einer Immobilie dem Eigentümer, sich einfach und unkompliziert liquide Mittel zu beschaffen, was wiederum die Kosten der Illiquidität reduziert. Beides wirkt sich steigend auf die Immobilienpreise aus.

Das Potenzial der Tokenisierung von illiquiden Vermögenswerten ist gross. Alleine der weltweit geschätzte Wert aller bestehenden Immobilien im Jahr 2016 betrug ca. 217 Billionen USD. ${ }^{1}$ Die Tokenisierung von Immobilien steht jedoch erst ganz am Anfang. Das erste Objekt, dessen Token unmittelbar nach der Emission auf einer öffentlich zugänglichen Plattform weltweit handelbar waren, wurde erst im Jahr 2019 tokenisiert. $^{2}$

Die Bundesanstalt für Finanzdienstleistungsaufsicht (BaFin) versteht unter Token eine digitalisierte Form von Vermögenswerten, der eine bestimmte Funktion oder ein bestimmter Wert zugesprochen wird. ${ }^{3}$ Ein virtueller Token repräsentiert somit ein „Etwas“ in einer Organisation, in einem System oder in einem Netzwerk. Dieses „Etwas“ kann einen Wert, einen Anteil, ein Stimmrecht oder eine anderweitige Form der Beteiligung darstellen. Ein virtueller Token kann auch mehrere Rollen gleichzeitig erfüllen, er kann beispielsweise sowohl einen Anteil an einem Netzwerk als auch ein Stimmrecht verkörpern. Der vorliegende Aufsatz beschränkt sich auf die virtuelle Repräsentation eines Wertes. Dieser Wert kann ein Anrecht auf einen Anteil am Cashflow einer Immobilie sein. Unter dem Begriff der Tokenisierung versteht man hierbei den Vorgang, der digitale Token schafft.

Der Fokus dieses Artikels liegt in der Anwendung der Tokenisierung im Immobilienbereich. Zu diesem Zweck führt der zweite Abschnitt in die Blockchain-Technologie ein. Der dritte Abschnitt gibt eine kurze Übersicht über die Klassifizierung und die Regulierung von Token. Die Tokenisierung von illiquiden Vermögenswerten und die Vorteile der Tokenisierung werden im vierten Abschnitt diskutiert. Im fünften Abschnitt dokumentieren wir die erste tokenisierte Immobilie, deren Token auch auf einer öffentlich zugänglichen Plattform gehandelt werden. Der letzte Abschnitt beinhaltet schließlich einen Ausblick darauf, wie diese neue Technologie die Finanz- und Immobilienmärkte transformieren könnte.

\section{Blockchain und ihre Eigenschaften}

Eine Blockchain ist eine verteilte Datenbank, die aus einer Kette von durch kryptografische Verfahren miteinander verbundenen Datenblöcken besteht und gleichzeitig auf mehreren Computern geführt wird. Die ersten Blockchains wurden bereits in den

\footnotetext{
1 Vgl. Savills Research (2016) abrufbar auf https://www.savills.com/research_articles/255800/198669-0. Gesehen am 23. März 2020.

2 Vgl. https://realt.co/product/9943-marlowe-st-detroit-mi-48227/. Gesehen am 09. September 2020.

3 Vgl. BaFin: https://www.bafin.de/dok/12342268. Gesehen am 03. Juli 2020.
} 
1990er-Jahren entwickelt und zwar mit dem Ziel, Aufzeichnungen manipulationsresistent zu machen. ${ }^{4}$

In der Praxis wird oft zwischen permissioned und nonpermissioned Blockchains unterschieden. Von einer nonpermissioned Blockchain spricht man, wenn es keine Zugangsbeschränkungen zum Netzwerk gibt. Jede Person kann an allen Aktivitäten des Netzwerks teilnehmen, ohne um Erlaubnis zu fragen. Gleichzeitig kann die Blockchain von allen Teilnehmern eingesehen und überprüft werden. Die gesamte benötigte Software für den Betrieb eines Knotens ${ }^{5}$ ist zudem öffentlich zugänglich (open source).

Eine nonpermissioned Blockchain hat den Vorteil, dass sie mit der Zeit immer robuster wird. Erstens werden Fehler in der Software schnell identifiziert, weil viele Entwickler mit unterschiedlichstem Hintergrund an der Weiterentwicklung der Software arbeiten. Zweitens wächst im Erfolgsfall das Netzwerk, weil immer mehr Knoten dazukommen. Die geschaffene Dezentralität führt dazu, dass es keine zentralen Angriffspunkte gibt.

Eine permissioned Blockchain ist eine zentralisierte Datenbank, in der eine Institution die Zugangsrechte verwaltet, wofür auch die Identität der Teilnehmer bekannt sein muss. ${ }^{6}$ Oft wird im Unternehmensbereich oder bei Regierungen argumentiert, dass eine permissioned Blockchain den Vorteil hat, dass die Daten nicht öffentlich einsehbar sind. Es ist jedoch auch möglich, auf einer öffentlichen Blockchain Daten verschlüsselt abzulegen. Der Artikel untersucht ausschließlich die Tokenisierung von Immobilien auf einer öffentlichen Blockchain. ${ }^{7}$

Die Eigenschaften der Blockchain werden anhand der weit verbreitenden BitcoinTechnologie erklärt. Da der/die Bitcoin-Erfinder, bekannt unter dem Pseudonym Satoshi Nakamoto (2008), eine virtuelle Währung bereitstellen wollte/n, die ohne zentrale Instanzen und Intermediäre funktioniert, mussten sie eine Technologie entwickeln, die es ermöglicht, eine Blockchain in einem offenen Computernetzwerk zu führen. Die Bitcoin-Blockchain ist nonpermissioned und wird simultan von tausenden Knoten aktualisiert und ermöglicht, dass ein virtueller Vermögenswert ohne zentrale Instanzen verwaltet werden kann.

Treffend beschreiben Berentsen und Schär (2017) die fundamentale Bedeutung der Bitcoin-Blockchain: „Erstmals ist es möglich, Besitzverhältnisse von virtuellem Eigentum eindeutig festzuhalten, ohne dass dafür eine zentrale Instanz benötigt wird - eine Entwicklung, die das Potenzial hat, die bestehende Zahlungsinfrastruktur und das Finanzsystem grundlegend zu verändern“. Die wichtigsten Eigenschaften der Bitcoin-Blockchain sind: Konsistenz der Daten, Unveränderbarkeit der Daten, Besitzbarkeit der Daten, Konsens und Pseudonymität.

\footnotetext{
${ }^{4}$ Eine kryptographisch gesicherte Kette von Blöcken wird zum ersten Mal von Haber und Stornetta (1991) beschrieben.

5 Ein Knoten in einem Computernetzwerk ist ein Computer der Daten empfängt, speichert und weiterleitet.

6 Vgl. Narayanan et al. (2016, S. 27-28).

7 Für zusätzliche Informationen zu den Vor- und Nachteilen von permissioned und nonpermissioned Blockchains vgl. beispielsweise https://www.blockchain-council.org/blockchain/advantages-anddisadvantages-of-permissionless-blockchain/. Gesehen am 22. Juli 2020.
} 


\subsection{Konsistenz der Daten}

Die Konsistenz der Datenbank wird dadurch erreicht, dass bei der Hinzufügung von Daten immer kontrolliert wird, dass die neuen Daten nicht im Widerspruch zu den bereits bestehenden Daten stehen. Dadurch werden sogenannte ,doublespends" verhindert, in denen ein Bitcoin-Guthaben mehrere Male ausgegeben wird. Dies bedeutet, dass jede neue Bitcoin-Zahlung daraufhin überprüft wird, ob die zu transferierenden Bitcoin-Einheiten nicht bereits zu einem früheren Zeitpunkt ausgegeben wurde. ${ }^{8}$

\subsection{Unveränderbarkeit der Daten}

Die Bitcoin-Blockchain ist eine ,append-only“-Datenbank, d.h. es lassen sich nur Daten hinzufügen, aber keine nachträglich ändern. Es ist an dieser Stelle nicht möglich, alle Elemente darzustellen, die die Unveränderbarkeit der Bitcoin-Blockchain ermöglichen. Dies sind einerseits kryptographische Methoden, andererseits aber auch ausgeklügelte Anreizsysteme. Eines der wichtigsten Elemente ist das die Bitcoin-Blockchain als verteilte Datenbank in einem offenen Netzwerk geführt wird. Dadurch gibt es keine spezielle Bitcoin-Blockchain, sondern nur Tausende von identischen Kopien, die simultan und unabhängig aktualisiert werden. ${ }^{9}$

\subsection{Besitzbarkeit der Daten}

Die Eigenschaft der „Besitzbarkeit“ der Daten ist eine der besonderen Eigenschaften der Bitcoin-Blockchain. Sie beruht auf der asymmetrischen Kryptographie. Der Eigentümer einer Bitcoin-Einheit verfügt über ein kryptographisches Schlüsselpaar: einen privaten und einen öffentlichen Schlüssel. Der öffentliche Schlüssel dient als Adresse, auf die Bitcoin-Einheiten überwiesen werden können. Mit dem privaten Schlüssel kann der Besitzer eines Coins dem Bitcoin-Netzwerk beweisen, dass er der rechtmässige Eigentümer dieser Einheit ist. Der Grund dafür ist, dass nur eine Person, die Zugang zum privaten Schlüssel hat, über diesen Guthaben verfügen kann. ${ }^{10}$ Man spricht davon, dass die asymmetrische Kryptographie einer Person ermöglicht, einen virtuellen Vermögenswert mittels kryptographischer Methoden zu beherrschen. ${ }^{11}$

\footnotetext{
8 Vgl. beispielsweise Antonopoulos (2017), Berentsen und Schär (2017) und Narayanan et al. (2016).

9 Vgl. beispielsweise Antonopoulos (2017), Narayanan et al. (2016) und Berentsen und Schär (2020).

${ }^{10}$ Ein Bitcoin ist teilbar. Die kleinste Einheit ist der Satoshi und ein Bitcoin kann in 10.000.000 Satoshis aufgeteilt werden.

11 Vgl. beispielsweise Antonopoulos (2017), Berentsen und Schär (2017) und Narayanan et al. (2016). Das bedeutet, dass ein Token sich mittels kryptografischen Methoden handeln, transferieren und verwalten lässt. Der Begriff „beherrschen“ vermittelt auch, dass niemand - ausser dem Besitzer des privaten Schlüssels - Zugriff zum Token hat.
} 


\subsection{Konsens über die Daten}

In der Bitcoin-Technologie bedeutet der Begriff „Konsens“, dass sich alle Teilnehmer an die Regeln halten, die im Bitcoin-Protokoll festgehalten sind. ${ }^{12}$ In einem dezentralen System kann aber kein Teilnehmer zur Einhaltung der Regeln gezwungen werden. Damit dieses funktioniert, müssen die Regeln derart ausgestaltet werden, dass es im Eigeninteresse jedes Teilnehmers ist, sich an diese zu halten. Ist dies der Fall, spricht man in der Sprache der Spieltheorie von einem Nash-Gleichgewicht (Nash 1951). Dieses beschreibt eine Strategiekombination, in der jeder Spieler eine Strategie wählt, welche eine beste Antwort auf die Strategiewahl seiner Kontrahenten ist. ${ }^{13}$

Die wichtigste Implikation des Konsenses im Bitcoin-System ist, dass sich alle Teilnehmer jederzeit über die Eigentumsverhältnisse einig sind. In einem traditionellen Zahlungssystem wird dieser Konsens erreicht, indem man die Verwaltung der Datenbank zentralisiert und letztendlich diese zentrale Instanz oder anschließend ein Gericht darüber entscheidet, wem etwas gehört. In der neuen Bitcoin-Welt lässt sich das ohne zentrale Akteure bewerkstelligen. Das ist eindeutig ein technologischer Durchbruch. ${ }^{14}$

\subsection{Pseudonymität}

Die Teilnehmer im Bitcoin-Netzwerk sind pseudonym, weil nur deren öffentliche Adressen bekannt sind..$^{15}$ Die Kenntnis der öffentlichen Adresse einer Person ist ausreichend, um mit ihr zu interagieren. Die Pseudonymität hat in der Anfangszeit dazu geführt, dass Bitcoin auch für die Finanzierung von illegalen Aktivitäten verwendet wurde. ${ }^{16}$

Die Bitcoin-Blockchain funktioniert seit mehr als zehn Jahren ohne nennenswerte Unterbrechungen, obwohl es keine Zugangsbeschränkungen gibt und die Teilnehmer pseudonym sind. Die Robustheit (im Englischen ,resilience“) des Bitcoin Systems

\footnotetext{
12 Die Regeln des Bitcoin-Protokolls wurden erstmals im Bitcoin-Whitepaper (Nakamoto 2008) formuliert. Das Bitcoin-Netzwerk wurde zu Beginn des Jahres 2009 gestartet. Die Bitcoin-Software wird laufend verbessert und dadurch auch marginal die Regeln des Bitcoin-Systems.

${ }^{13}$ Es ist möglich, dass externe Akteure mit nicht-monetäre Anreizen das Bitcoin-System angreifen und schädigen können, indem sie eine Mehrheit an den Computerressourcen erwerben, um die Bitcoin-Blockchain zu dominieren. Dies würde unmittelbar den Wert der Bitcoin-Einheiten reduzieren und ein solcher Angriff wäre ökonomisch nicht rentable. Die Beschreibung des Nash-Gleichgewichts beschränkt sich auf Akteure, die ausschliesslich monetäre Ziele verfolgen.

14 Vgl. beispielsweise Antonopoulos (2017), Berentsen und Schär (2017) und Narayanan et al. (2016).

15 Bei der Pseudonymisierung wird die Identität einer Person durch ein Pseudonym mit dem Ziel ersetzt, die Identifikation der betroffenen Person zu verhindern.

${ }^{16}$ Ein gutes Beispiel für die Verwendung von Bitcoin-Zahlungen in der Grauzone ist der virtuelle Schwarzmarkt Silk Road, die im Februar 2011 online gestellt und zwei Jahre später geschlossen wurde. Silk Road ermöglichte den anonymen Handel mit beliebigen, oft illegalen Waren und Dienstleistungen. In der Zwischenzeit ist die Erkenntnis gewachsen, dass der Gebrauch von Bitcoin zur Finanzierung von illegalen Aktivitäten äußerst riskant ist. Der Grund dafür ist, dass alle Transaktionen auf einer öffentlichen Datenbank gespeichert werden und die forensischen Methoden immer besser werden, die die Verlinkung von öffentlichen Adressen und Identitäten ermöglichen.
} 
hat zur Folge, dass sich immer mehr Unternehmen und Regierungen mit den Vorund Nachteilen dieser Technologie auseinandersetzen. ${ }^{17}$

Es gibt jedoch auch viele Herausforderungen wie die mangelnde Skalierbarkeit, die Instabilität des Bitcoin-Preises und regulatorische Hemmnisse, die einer breiten Anwendung im Wege stehen. ${ }^{18}$ Zudem wird der Energieverbrauch des proof-of-work Konsensus-Mechanismus kritisiert. ${ }^{19}$

\section{Regulierung der Tokenisierung}

Die technische Umsetzung der Tokenisierung einer Immobilie ist verhältnismässig einfach und wird im vierten Abschnitt beschrieben. Regulatorische Unsicherheiten beschränken jedoch zurzeit noch eine breite Anwendung dieser Technologie. Unter dem Begriff „regulatorische Unsicherheit“ versteht man die Unsicherheit, die entstehen kann, wenn die Finanzmarktregulatoren noch keine umfassenden Rechtsvorschriften ausgearbeitet haben, auf die man sich als Token-Emittent oder Investor berufen kann. ${ }^{20}$ In diesem Abschnitt diskutieren wir die rechtlichen Aspekte, die bei einer Tokenisierung einer Immobilie berücksichtigt werden müssen.

\subsection{Beschreibung des herkömmlichen Kapitalmarktes}

In den meisten Ländern unterscheidet der Gesetzgeber bei der Regulierung des Kapitalmarkts zwischen professionellen Anlegern und Privat- oder Kleinanlegern. In Deutschland gehören beispielsweise zur Gruppe der professionellen Anleger Investoren, die ,über ausreichende Erfahrungen, Kenntnisse und Sachverstand verfügen, um ihre Anlageentscheidungen zu treffen und die damit verbundenen Risiken angemessen beurteilen zu können. " ${ }^{21}$ Professionelle Anleger haben einen direkten Zugang zum Kapitalmarkt.

Für die Mitgliedstaaten der Europäischen Union sind die Kriterien für professionelle Investoren in der Richtlinie des Europäischen Parlaments 2014/65/EU (Anhang II) festgelegt. ${ }^{22}$ Die nachstehende Aufzählung umfasst alle zugelassenen Rechtssubjekte, die von einem Mitgliedstaat im Rahmen dieser Richtlinie zugelassen oder beaufsichtigt werden: Kreditinstitute, Wertpapierfirmen, sonstige zugelassene oder beaufsichtigte Finanzinstitute, Versicherungsgesellschaften, Organe für gemeinsame Anlagen und ihre Verwaltungsgesellschaften, Pensionsfonds und ihre Verwaltungsgesellschaften, Warenhändler und Warenderivate-Händler, örtliche Anleger, sonstige institutionelle Anleger.

\footnotetext{
17 Fast $60 \%$ von 800 befragten Führungskräften und Experten prognostizieren, dass bis zum Jahr 2025 etwa zehn Prozent des globalen GDP von der Blockchain Technologie tangiert werden (WEF 2015).

18 Vgl. Antonopoulos (2017), Berentsen und Schär (2017) und Narayanan et al. (2016). Weiterführende Literatur: Diffie und Hellman (1976), Antonopoulos und Wood (2018), Berentsen und Schär (2020).

19 Vgl. de Vries (2018).

20 Vgl. weiterführende Literatur: Voshmgir (2019a, b).

21 Vgl. Richtlinie 2014/65/EU (Anhang II) und dejure (2020) § 67 Abs. 2 Satz 1 WpHG.

22 Vgl. EU (2014b), gesehen am 02. Juli 2020.
} 
Im Gegensatz zur Gruppe der professionellen Anleger haben Kleinanleger keinen direkten Zugang zum Kapitalmarkt. Sie sind auf sogenannte Finanzintermediäre angewiesen, wodurch ihnen indirekt die Marktpartizipation ermöglicht wird. Deren Rolle liegt darin, die Kleinanleger mit den notwendigen Informationen und den Finanzprodukten zu versorgen und das Vertrauen der Anleger in diese Märkte zu stärken. Das Kapitalmarktrecht ${ }^{23}$ hat den Zweck, die Anleger wirksam zu schützen und die Stabilität des Finanzsystems zu sichern. Daraus lassen sich die Hauptaufgaben einer Finanzaufsichtsbehörde ableiten, die bislang hauptsächlich darin bestanden, Intermediäre wie Kreditinstitute zu beaufsichtigen.

Der Grundsatz der Finanzmarktaufsichtsbehörden in vielen Ländern fordert unter anderem die Finanzgeschäfte technologieneutral $\mathrm{zu}$ betrachten und $\mathrm{zu}$ regulieren. ${ }^{24}$ Das bedeutet auch, dass die Finanzprodukte mit vergleichbaren Risiken identisch reguliert werden sollen. Die Regulierung der blockchain-basierten Finanzinstrumente wie Token hängt daher in erster Linie von deren Eigenschaften und Risiken ab. Da jeder Token frei programmierbar ist und deshalb andere Funktionen und Eigenschaften annehmen kann, besteht noch keine allgemein rechtlich anerkannte Klassifizierung von Token. ${ }^{25}$ Die BaFin selbst definiert einen (Krypto-)Token wie folgt: „Kryptotoken sind digitalisierte, auf einer Blockchain dezentral gespeicherte Abbildungen von Vermögenswerten“". ${ }^{26}$ Im Folgenden wird eine aufsichtsrechtliche Einordnung verschiedener Untergruppen von Token vorgestellt.

\subsection{Klassifizierung von Token}

(i) Payment-Token sind auch bekannt als Zahlungs- oder Krypto-Währungs-Token dabei handelt es sich um Token, die primär als Zahlungsmittel für Transaktionen zwischen den Nutzern eingesetzt werden und bereits übertragbar sind.

(ii) Utility-Token (Nutzungs-Token) stellen eine Art virtuellen Gutschein dar, der den Inhabern einen funktionalen Nutzen in Form eines Zugangs zu einem Netzwerk vermitteln sollen. Diese Token können besondere Rechte mit sich bringen, so beispielsweise die Möglichkeit, die Token zu einem späteren Zeitpunkt gegen Produkte oder Dienstleistungen eintauschen zu können.

(iii) Security-Token ${ }^{27}$ sind Finanzinstrumente, die ein Zahlungsversprechen beinhalten. Dadurch ähneln sie einer Aktie oder einer Obligation und sind daher mit her-

\footnotetext{
${ }^{23}$ Es existiert keine allgemein gültige Kodifizierung des Kapitalmarktrechts, sondern es gibt verschiedene Schriftstücke wie Gesetze, Verordnungen, Satzungen, Leitfaden, Rundschreiben, Richtlinien usw., die in großen Teilen nicht nur das Aufsichtsrecht, sondern teilweise auch zivil- und strafrechtliche Belange sowie einzelne Bereiche des Kapitalmarktrechts regeln.

24 BaFin Perspektiven (2018, S. 53 ff.).

25 Die deutsche Bundesanstalt für Finanzdienstleistungsaufsicht (BaFin) und die Schweizerische Finanzaufsichtsbehörde (FINMA) hat diese Klassifizierung jeweils in BaFin (2019) und FINMA (2018) ICOs guidelines festgelegt.

26 Fußwinkel und Kreiterling (2018).

27 Am 31. Januar 2019 hat die BaFin den ersten Security-Token mit Wertpapierprospekt für Privatanleger gebilligt und signalisierte damit, dass eine aufsichtskonforme Ausgabe von Token möglich ist.
} 
kömmlichen Wertpapieren nach Art. 4 Abs. 1 Nr. 44 der Richtlinie 2014/65/EU $\mathrm{zu}$ vergleichen. ${ }^{28}$

Damit ein Finanzinstrument als eine Security (Wertpapier) im Sinne des $\S 2$ Nr. 1 WpPG klassifiziert werden kann, bedarf es erstens der Übertragbarkeit, zweitens der Handelbarkeit am Finanzmarkt und drittens der Ausstattung mit wertpapierähnlichen Rechten. Ein Security-Token, der diese Eigenschaften erfüllt, ist durch vereinfachte Übertragbarkeit und erhöhte Handelbarkeit ausgezeichnet, jedoch mit dem Unterschied, dass er keine Verbriefung in Form einer Urkunde erfordert. ${ }^{29}$

Die Token-Kategorisierung von (i)-(iii) wird in erster Linie zu Vereinfachungszwecken verwendet und impliziert keine bindende Bewertung. Das führt dazu, dass in der Praxis jeder Token weiterhin unabhängig von seiner Form oder seiner Bezeichnung stets inhaltlich geprüft und bewertet werden muss. Häufig weisen Token hybride Formen auf, das heißt, die Utility- oder Security-Token dienen auch als Zahlungsmittel innerhalb ihres Netzwerkes. Folglich sind diese auch als PaymentToken zu betrachten. ${ }^{30}$

\subsection{Prospektpflicht für Security-Token}

Im europäischen Wertpapierrecht fallen unter die Kategorie der typischen Finanzinstrumente alle übertragbaren Wertpapiere - mit Ausnahme von Zahlungsinstrumenten - mit den folgenden Eigenschaften: Übertragbarkeit, Handelbarkeit und Standardisierung. ${ }^{31}$ Die Vorschriften des europäischen Wertpapierrechts legen fest, dass Wertpapiere mit einem Versprechen auf finanziellen Rendite als regulierte Wertpapiere im Sinne des Art. 4 Abs. 1 Nr. 44 der Richtlinie 2014/65/EU klassifiziert werden müssen. Darunter fallen in der Regel die Token, die bei einer Tokenisierung eines Vermögenswertes geschaffen werden. ${ }^{32}$ Dies hat diverse Folgen für Emission und Handel. Insbesondere sind die Emittenten eines solchen Token verpflichtet, öffentlich einen Prospekt vorzulegen.

Diese Prospektpflicht wird nun am Beispiel des deutschen Wertpapier-Prospektgesetzes (WpPG) dargestellt. Denn wenn ein Prospekt durch die deutsche Aufsichtsbehörde der Bundesanstalt für Finanzdienstleistungsaufsicht (BaFin) gebilligt wur-

\footnotetext{
28 Vgl. Directive 2014/65/EU of the European Parliament and of the Council of 15 May 2014 on markets in financial instruments and amending Directive 2002/92/EC and Directive 2011/61/EU Text with EEA relevance (EU 2014a).

29 Security-Token werden als ,tokenisierte“ Wertpapiere bezeichnet und deshalb sind wie prospektpflichtige Wertpapiere zu behandeln. Die Regulierungskompetenzen für solche FI werden in den Vereinigten Staaten der Securities and Exchange Commission (SEC), in der Schweiz der FINMA und in Deutschland der BaFin zugesprochen.

30 Vgl. BaFin (2019a, b).

31 Vgl. Art. 4 Abs. 1 Nr 44 der Richtlinie 2014/65/EU.

32 Werden finanzielle Zuwendungen wie etwa die Zahlung von Zinsen und Erträgen oder eine Beteiligung an dem Geschäftsergebnis des Emittenten mit dem Token verknüpft, handelt es sich um sogenannte Security-Token. Der Emittent von Security-Token muss in diesem Zusammenhang zu beachtende gesetzliche Bestimmungen im Blick haben. So handelt es sich in Deutschland bei verbrieften Kapitalmarktprodukten insbesondere um die Regelungen des Wertpapierprospektgesetzes (WpPG) und der europäischen Prospektverordnung (ProspektVO), die genaue Vorgaben für den zu erstellenden Wertpapierprospekt beschreiben.
} 
de, kann der Emittent das Wertpapier in der EWR-Staaten anbieten (,Europäischer Pass“). Um einen Security-Token auf einem organisierten Markt ${ }^{33}$ beispielsweise an einer Krypto-Börse (Crypto-Exchange) oder an einer anderen Handelsplattform (z.B. MTF $^{34}$ oder $\mathrm{OTF}^{35}$ ) als Security-Token ähnlich wie ein Wertpapier ${ }^{36}$ öffentlich anzubieten ${ }^{37}$ und zum Handel ${ }^{38}$ zuzulassen, muss der Emittent vor der Zulassung seiner Emission einen von der Aufsichtsbehörde ${ }^{39}$ gebilligten Prospekt veröffentlichen. Ein Verstoß dagegen stellt eine Ordnungswidrigkeit dar, die zu einer sofortigen Untersagung des Angebots durch die BaFin führt. ${ }^{40}$

Ein Prospekt ist eine Mitteilung an das Publikum, die ausreichende Informationen in leicht analysierbarer und verständlicher Form über das Wertpapier enthält. Die Bestandteile eines Prospektes sind in der Prospektverordnung (ProspVO 2017, 2019) geregelt. ${ }^{41}$ Insbesondere muss ein Wertpapierprospekt die folgenden Angaben enthalten:

- Darstellung und Erläuterung von Risikofaktoren, die für den Emittenten und seine Branche spezifisch sind bzw. seine Fähigkeit zur Erfüllung der Pflichten gegenüber den Anlegern beeinträchtigen können.

- Darstellung der Geschäfts- und Finanzlage (Operating und Financial Review), Pro-Forma-Angaben auf der Basis historischer Daten zum Emittenten, Prognosen über die künftige Geschäftsentwicklung.

- Erklärung zum Ausreichen des Geschäftskapitals für die derzeitigen Bedürfnisse und Angaben über die Beschaffung künftig notwendigen Kapitals.

- Angaben über die an der Geschäftsführung beteiligten Personen, Funktionsweise der Wertpapiere.

Der Prospekt besteht aus einer Zusammenfassung, einem Registrierungsformular und einer Wertpapierbeschreibung. Bei der Zusammenfassung handelt es sich um ein Dokument, das auf maximal sieben DIN-A4-Seiten prägnant und gut leserlich das Angebot beschreibt. Generiert die Tokenisierung von Vermögenswerten einen Token mit den Eigenschaften eines Security-Tokens und wird öffentlich angeboten,

\footnotetext{
33 Der Ausdruck organisierter Markt ist definiert in $\S 2$ Nr. 1 WpPG.

34 Definition zu MTF (Mulitlateral Trading Facility) ist in Art. 4 Abs. 1 Nr 22 der Richtlinie 2014/65/EU.

35 Definition zu OTF (Organised Trading Facilitiy) ist in Art. 4 Abs. 1 Nr 23 der Richtlinie 2014/65/EU.

36 Der Begriff Wertpapier ist definiert in $\S 2$ Nr. 1 WpPG.

37 Der Ausdruck öffentliches Angebot ist definiert in $\S 2$ Nr. 1 WpPG.

38 Der Ausdruck Zulassung zum Handel ist definiert in $\S 1$ Abs. 1 WpPG.

39 In Europa gibt es keine einheitliche Institution, die allein die Finanzmarktaufsicht übernimmt, sondern jeder Mitgliedstaat hat die Verantwortung für die Überwachung seines eigenen Marktes und verfolgt dabei seine eigene Regulierungsphilosophie. In Deutschland liegt diese Pflicht bei der Bundesanstalt für Finanzdienstleistungsaufsicht (BaFin), in der Schweiz bei Eidgenössische Finanzmarktaufsicht (FINMA), in Liechtenstein die Finanzmarktaufsicht Liechtenstein (FMA) usw.

40 Vgl. § 26 Abs. 4 Satz 1 WpPG.

41 Vgl. Art. 6 Abs. 1 ProspVO 2017. Ausnahmen von der Prospektpflicht oder eine gestufte Prospektpflicht bei geringwertigen Angeboten sind im Art. 3 WpPG regelt. Die Mindestangaben eines Prospektes, die dem Anlegerschutz dienen, sind im Art. 13 ProspVO 2017 festgelegt.
} 
ist diese mit der Verbriefung von Vermögenswerten vergleichbar. ${ }^{42}$ In diesem Fall sind die Prospektanforderungen für diesen Token unabdingbar. ${ }^{43}$

\section{Theorie: Tokenisierung von Immobilien}

Aus aufsichtsrechtlicher Perspektive sind die Verbriefung und die Tokenisierung von Vermögenswerten äquivalent. Der wesentliche Unterschied liegt in der Infrastruktur und der Technologie für die Emission und dem Handel mit diesen neuen Finanzinstrumenten. In diesem Abschnitt werden die wesentlichen technologischen Aspekte der Tokenisierung vorgestellt und die Vorteile der Tokenisierung hervorgehoben. Dabei wird der Fokus auf die Ethereum-Blockchain gelegt, die von Buterin (2013) konzipiert wurde, da sich diese als Standard für die Herausgabe von Token etabliert hat. ${ }^{44}$

Bei der Tokenisierung stückelt man den Wert eines illiquiden Vermögenswertes in untereinander austauschbare und handelbare virtuelle Token. ${ }^{45}$ Die Eigentumsrechte an diesen Token werden auf der Ethereum-Blockchain festgehalten und sie können auf dezentralisierten Handelsplätzen Peer-to-Peer oder auf zentralisierten KryptoBörsen gehandelt werden. Der Begriff Peer-to-Peer oder P2P bedeutet, dass alle Computer, die am Netzwerk teilnehmen, direkt miteinander kommunizieren können. Sie sind alle gleichwertig und teilen die Last der Bereitstellung von Netzwerkdiensten untereinander auf. ${ }^{46}$ Mit einer P2P-Zahlung wird ein Token-Betrag direkt zwischen zwei Peers ausgetauscht, ohne einen Intermediär dazwischenzuschalten. ${ }^{47}$

Ein wesentlicher Unterschied zwischen einem traditionellen Wertpapier und einem Security-Token ist die Verwaltung der Eigentumsrechte der Token. Ein Token ist mittels kryptographischer Methoden beherrschbar. Der Eigentümer eines Tokens und nur er selbst kann den Token transferieren, indem er eine Transaktionsnachricht erstellt, mit seinem privaten Schlüssel signiert und anschließend an das Ethereum Netzwerk versendet. Der Transfer von Token ist zudem ohne Intermediäre jederzeit möglich - also rund um die Uhr - da die Blockchain keine Öffnungszeiten kennt und sich weltweit auf dieses öffentliche Netzwerk zugreifen lässt. Im Gegensatz dazu ist der Handel eines traditionellen Wertpapiers nur über Finanzintermediäre möglich.

\footnotetext{
42 Im Artikel von Jobst (2008) versteht man unter einer Verbriefung (securitization auf Englisch) die Ausstellung von handelbaren Wertpapieren, die häufig durch illiquide Vermögenswerte gesichert werden. Dazu wird in der Regel eine Zweckgesellschaft gegründet, deren einzige Aufgabe die Herausgabe dieses Wertpapiers ist. Auf der Aktivseite der Bilanz sind die eingebrachten illiquiden Vermögenswerte und auf der Passivseite die emittierten Wertpapiere aufgeführt.

43 Vgl. BaFin (2019b).

44 Die Ausgabe von Token ermöglicht auch die Finanzierung von Unternehmen (Nyffenegger und Schär, 2018). Andere Plattformen, die eine Tokenisierung von Vermögenswerten erlauben, sind unter anderem EOS, Tezos und Cardano.

45 Die Literatur zu Kryptoassets unterscheidet zwischen Token und Coin. Ein Coin ist die native Währung einer Plattform (z. B. der Bitcoin für die Bitcoin-Blockchain und der Ether für die Ethereum-Blockchain). Ein Token wird auf einer bestehenden Plattform ausgegeben. Die meisten Token wurde auf der EthereumPlattform emittiert.

46 Vgl. Antonopoulos (2017).

47 Vgl. Nakamoto (2008).
} 


\subsection{Smart Contract}

Der Begriff „Smart Contract“ geht auf Nick Szabo (1996) zurück. Er beschreibt die Idee, dass vertragliche Verpflichtungen, wie beispielsweise ein Zahlungsversprechen, nicht auf einem Stück Papier, sondern als Code in einem Computernetzwerk festgehalten und dann autonom umgesetzt werden, wenn gewisse Bedingungen erfüllt werden. Seit der Einführung der Bitcoin-Technologie im Jahr 2009 wird die Idee eines "Smart Contract“" mit der Blockchain-Technologie in Verbindung gebracht. Automatisierte Prozesse sind jedoch im traditionellen Finanzsektor und anderen Bereichen der Wirtschaft schon seit Jahrzenten Gang und Gäbe. Dafür braucht es keine Blockchain-Technologie. Die Blockchain-Technologie erlaubt einzig und alleine, dass ein Smart Contract, wenn er auf einer dezentralen Plattform wie Ethereum bereitgestellt wird, zensurresistent ist, da er nachträglich nicht veränderbar ist..$^{48}$

Die Ethereum-Blockchain wurde vor fünf Jahren ins Leben gerufen und hat sich sehr schnell als wichtigste nonpermissioned Blockchain für Smart Contracts etabliert. Die meisten Smart Contracts werden heute mit der Programmiersprache Solidity entwickelt. Sie erlaubt die Entwicklung von beliebig komplexen Smart Contracts. Zudem kann ein Smart Contract mit anderen auf der Ethereum Plattform bereitgestellten Smart Contracts interagieren.

Nach der Entwicklung eines Smart Contracts wird er auf der Ethereum-Blockchain zur Nutzung bereitgestellt. Im Zuge der Bereitstellung erhält er eine eindeutige Ethereum-Adresse. Ein Nutzer, der mit einem Smart Contract interagieren möchte, sendet in der Regel eine Transaktionsnachricht an diese Adresse. Alle Informationen und Transaktionen, die innerhalb eines Smart Contracts stattgefunden haben, sind öffentlich und z.B. auf Etherscan ${ }^{49}$ unter der Smart Contract-Adresse einsehbar.

\subsection{Technologie der Tokenisierung}

Auf der Ethereum-Plattform lassen sich Token als Smart Contracts nach unterschiedlichen Standards emittieren. Die meisten Token halten sich an den sogenannten ERC-20 Token Standard. ${ }^{50}$ Die wichtigste Funktion eines Smart Contracts für die Tokenisierung ist die Verwaltung des Eigentums an den herausgegebenen Token. Durch eine Transaktionsnachricht kann die an einem Token berechtigte öffentliche Ethereum-Adresse verändert werden.

Aus der Sicht der Nutzer erfordert der Besitz und der Transfer eines Tokens eine kompatible Wallet. Dabei handelt es sich um eine Softwareanwendung, die primär den unkomplizierten Empfang und Versand von Token erlaubt. Für einen Eigentumsübertrag eines Tokens verfasst der Sender mit Hilfe der Wallet eine Transaktionsnachricht. Neben anderen Informationen beinhaltet die Nachricht die Adresse des Empfängers des Tokens, die Kontraktadresse und die Anzahl der Token, die transferiert werden sollen. Ein solcher Eigentumsübertrag ist in der Regel in we-

\footnotetext{
48 Vgl. Berentsen und Schär (2020).

$49 \mathrm{Vgl}$. Etherscan auf https://etherscan.io/. Gesehen am 02. Juli 2020.

$50 \mathrm{Vgl}$. Buterin und Vogelstellert (2015). Github auf https://github.com/ethereum/EIPs/blob/master/EIPS/ eip-20.md. Gesehen am 02. Juli 2020.
} 
nigen Sekunden nach der Versendung der Transaktionsnachricht in der EthereumBlockchain registriert.

Bei der Gestaltung eines Tokens müssen verschiedene Parameter geregelt werden. Die wichtigsten Parameter sind zum Beispiel die Anzahl der Token, die geschaffen werden, die Anzahl der Token, die zum Verkauf angeboten werden, der Ausgabepreis eines Tokens, die Teilbarkeit des Tokens, die akzeptierten Zahlungsmittel beim Verkauf, das Verkaufsfenster sowie das Berücksichtigen eventueller Handelsrestriktionen.

Wenn die Tokenisierung auf der Ethereum-Blockchain stattfindet, wird in der Regel der Ether als Zahlungsmittel akzeptiert. Oft erlaubt der Emittent auch eine Zahlung in einer staatlichen Währung. In jedem Fall muss der Käufer eine Ethereum Adresse bereitstellen, auf der die erworbenen Token gutgeschrieben werden können.

Möchte ein Emittent die Handelbarkeit eines Tokens beschränken, kann er dies auch über einen Smart Contract regeln. Dazu erstellt er eine sogenannte Whitelist, die alle öffentlichen Adressen, mit der Befugnis, Token zu erhalten, aufführt. Der Smart Contract, welcher das Eigentum an den Token verwaltet, lässt dann nur Transaktionen zwischen Adressen zu, welche in der Whitelist aufgeführt sind.

Je nach Land müssen die Handelbarkeit eines Tokens unterschiedliche regulatorische Vorschriften erfüllen. Da bei der Tokenisierung einer Immobilie die emittierten Token als Security-Token eingestuft werden, kommen die entsprechenden gesetzlichen Bestimmungen, wie Know-Your-Customer-Auflagen (KYC) zur Anwendung. Die Erfüllung dieser Auflagen erfordert einen aufwendigen Registrierungsprozess, der mit den KYC-Auflagen bei einer Kontoeröffnung im klassischen Finanzsystem vergleichbar ist. Dazu gehören Wohnsitz- und Ausweiskontrollen sowie entsprechende Maßnahmen zur Vermeidung von Geldwäscherei. ${ }^{51}$

\subsection{Vorteile der Tokenisierung}

In diesem Abschnitt diskutieren wir die Vorteile der Tokenisierung einer Immobilie. Dabei besprechen wir die Kosten, Liquidität, Standardisierung, Flexibilität, Transparenz und Sicherheit.

\subsubsection{Emissionskosten und Transaktionskosten}

Zum aktuellen Zeitpunkt befindet sich die Tokenisierung von illiquiden Vermögenswerten oder Immobilien noch in den Kinderschuhen. Der Aufwand ist gross, um alle regulatorischen Vorschriften zu erfüllen. Dadurch ist die Kapitalbeschaffung über eine Tokenisierung im Vergleich zu einer traditionellen Kapitalbeschaffung nicht zwingend günstiger. Die Kosten der Tokenisierung von einer Immobilie werden im fünften Abschnitt anhand eines Fallbeispiels detailliert aufgezeigt.

Diese Kosten werden jedoch aus zwei Gründen schnell sinken. Erstens wird die rechtliche Unsicherheit mittelfristig abnehmen. In der Schweiz wurde beispielsweise bereits im Jahr 2019 Gesetzesänderungen vorgeschlagen, die viele dieser rechtlichen Hürden beseitigen sollten. Im Juni 2020 hat dann der Schweizerische Nationalrat

51 Vgl. Berentsen und Schär (2017). 
einstimmig diese Änderungen angenommen. Das neuen Schweizerische DLT-Gesetz hat das Ziel, die Rechtssicherheit zu erhöhen, Hürden für Blockchain-Anwendungen zu beseitigen und Missbrauchsrisiken zu begrenzen. ${ }^{52}$ Dieses Beispiel kann interpretiert werden, dass sich die Schweiz als attraktiver Standort für die Distributed Ledger Technologie (DLT) positionieren möchte.

Zweitens können viele notwendige Schritte der Tokenisierung standardisiert und automatisiert werden. Der ERC20-Token-Contract ist open source und kann deshalb legal kopiert und angepasst werden. Auch die benötigten juristischen Dokumente für eine Tokenisierung werden mittelfristig standardisiert und günstig erhältlich sein. Dadurch werden mit der Zeit immer weniger Intermediäre (Banken, Anwälte, Makler usw.) benötigt und langfristig die Kosten der Kapitalbeschaffung über eine Tokenisierung stark sinken.

\subsubsection{Liquidität}

Einer der großen Vorteile der Tokenisierung ist der einfache Sekundärhandel. Jeder ERC20-Token ist theoretisch unmittelbar nach der Ausgabe weltweit handelbar und zwar rund um die Uhr. Dazu muss er einfach auf einer der vielen dezentralisierten Handelsplätze wie beispielsweise Uniswap oder Kyber für den Sekundärhandel aktiv werden. Auf diesen dezentralisierten Börsen kann jeder Token gelistet und gehandelt werden, ohne dass eine zentrale Instanz um Erlaubnis gefragt werden muss. Die Registrierung kann zudem durch irgendeine Person getätigt werden. Ein Käufer braucht dann nur eine ERC20-Token kompatible Wallet um den Token im Sekundärhandel zu erwerben. ${ }^{53}$

Heute werden die meisten Kryptowährungen auf zentralisierten Krypto-Börsen wie Coinbase, Binance oder Bittrex gehandelt. Diese zentralisierten Handelsplätze nehmen einen neuen Token in den Handel auf, wenn er einen hinreichend großen Umsatz verspricht und regulatorisch unbedenklich ist. Die meisten ImmobilienToken dürften ein solches Umsatzkriterium nicht erfüllen.

\subsubsection{Standardisierung}

Die Emission eines ERC20-Tokens ist technisch einfach. Es gibt Vorlagen für Smart Contracts, die in kürzester Zeit auf die eigenen Bedürfnisse angepasst werden können. Dadurch wird die Zeit zwischen der Entscheidung, eine Immobilie zu tokenisieren, und der Herausgabe des Tokens stark reduziert. Die sich abzeichnende Standardisierung wird es in der Zukunft ermöglichen, weltweit über Token Eigentum an Immobilien zu erwerben, die mit dem gleichen Standard geschaffen wurden.

Darüber hinaus wird die Standardisierung die Suche nach tokenisierten Immobilien langfristig vereinfachen. Es werden Plattformen entstehen, die diese Liegenschaften aus allen Teilen der Welt zugänglich machen und diese nach vergleichbaren Kriterien sortieren.

\footnotetext{
52 Vgl. Wälti (2020).

53 Der Eigentumsübertrag auf der Ethereum-Blockchain ist in wenigen Sekunden abgewickelt. In der traditionellen Finanzwelt kann ein Eigentumsübertrag an einem Wertpapier mehrere Tage dauern.
} 


\subsubsection{Flexibilität}

Solidity, die Programmiersprache der Ethereum-Blockchain, ist äußerst flexibel. Sie erlaubt es, theoretisch einen beliebig komplexen Smart Contract zu schreiben. Dadurch lassen sich viele Prozesse automatisieren, wie später im praktischen Teil bei der Tokenisierung einer Immobilie näher beschrieben wird. In diesem Beispiel erwirbt ein Token-Besitzer ein Anrecht auf einen Teil der Mieteinnahmen der Immobilie. Die Auszahlungen an die Token-Besitzer werden durch den Smart Contract automatisiert.

Smart Contracts erlauben, dass Auszahlungen an gewisse Bedingungen geknüpft werden wie beispielsweise eine Halteperiode. Denkbar sind auch monatliche, wöchentliche oder sogar tägliche Auszahlungen. Diese Zahlung kann mittels der nativen Währung der Ethereum-Plattform (Ether) erfolgen oder auch mit einem Stablecoin wie dem DAI. ${ }^{54}$

\subsubsection{Sicherheit und Transparenz.}

Die Ethereum-Blockchain ist eine öffentliche Datenbank, die für jedermann einsehbar ist. Dadurch kann auch jeder einen Smart Contract unter die Lupe nehmen, um herauszufinden, ob er die vertraglichen Verpflichtungen der Herausgeber auch erfüllt. Eine solche Inspektion ist allerdings nur für erfahrene Programmierer zu bewältigen. Inzwischen gibt es immer mehr Unternehmen, die sich auf die Audits von Smart Contracts spezialisiert haben.

Ein weiterer, bisher noch nicht genannter Aspekt hinsichtlich den Anwendungsmöglichkeiten der Blockchain ist der Manipulationsschutz von Dokumenten. Dazu wird der Hashwert eines Dokuments - zum Beispiel ein Kaufvertrag - berechnet und auf der Ethereum-Blockchain abgespeichert. Da jegliche Änderung des Dokuments unweigerlich dessen Hashwert verändert, wird eine Modifikation des Dokuments sofort erkannt werden. Da es fast unmöglich ist, die Ethereum-Blockchain nachträglich zu ändern, kann ein Fingerabdruck als Beweis dafür dienen, dass eine bestimmte Datei zu einem bestimmten Zeitpunkt existierte und das Dokument in der Zwischenzeit nicht verändert wurde.

\section{Praxis: Tokenisierung von Immobilien (Realtoken)}

Die RealToken ${ }^{55} \mathrm{GmbH}$, mit dem Sitz in Aventura, Florida, USA, ist eine eingetragene Gesellschaft mit beschränkter Haftung, die von der RealToken Inc. verwaltet wird. Der allererste Token, der von der RealToken GmbH im Jahr 2019 emittiert

\footnotetext{
54 En Stablecoin ist eine Kryptowährungen, die eine geringe Preisvolatilität aufweist. Der DAI ist beispielsweise eine Kryptowährung, welche 1:1 an den USD gekoppelt ist (Berentsen und Schär 2019).

55 Vgl. RealT (2019b). Eine Mischung aus dem Wort Reality und Token ergibt den Namen des Projektes RealT oder RealToken. Vgl. die offizielle Webseite unter https://realt.co/ und https://realt.co/wp-content/ uploads/2019/05/RealToken_White_Paper_US_v03.pdf. Gesehen am 14. Juni 2020.
} 
wurde, heißt „RET-9943-MARLOWE-STREET-MI““.56 Tokenisiert wurde ein Einfamilienhaus, das unter der Adresse 9943 Marlowe St, Detroit, MI 48227 auffindbar ist. $^{57}$

Der Preis der Immobilie betrug 57.300 USD. Es wurden eintausend Token zum Preis von 63,75 USD pro Token herausgegeben und verkauft. Die Einnahmen betrugen 63.750 USD. Die Token werden zur Zeit im Sekundärhandel auf der Uniswap Plattform zum Preis von 76,73 USD gehandelt. ${ }^{58}$

Der Token wurde auf der Grundlage des ERC20 Token-Standards entwickelt. Mit dem Kauf eines Tokens erwirbt man ein Anrecht auf einen Teil der Mieteinnahmen. Die jährliche Zahlung beträgt zurzeit 7,9 USD. Gemäß der Auskunft der Webseite von RealToken $\mathrm{GmbH}$ entspricht dieser Betrag einer Rendite abzüglich Steuern und Verwaltungskosten von 13,0\%. Alle Auszahlungen erfolgen mittels des Stablecoins DAI.

Potenzielle Kostenersparnisse werden oft als ein Vorteil der Tokenisierung genannt. Es ist jedoch viel zu früh, um zum Thema Kostenersparnis ein definitives Urteil zu fällen. In unserem Beispiel betrugen die Emissionskosten ungefähr zehn Prozent. ${ }^{59}$ Die Interpretation ist jedoch schwierig, da alle Kosten unter dem Kostenträger „Working Capital“ subsumiert wurden, wie Abb. 1 aus dem offiziellen Dokument zeigt. ${ }^{60}$

In diesem Dokument wird auch erwähnt, dass die Bankgebühren (Investment Banking Advisor Fees) Null USD betragen haben. Im Kleingedruckten steht, dass es eine ,,5 \% Cash Service Fee“-Gebühr von 3187,5 USD gegeben hat, die der Verkäufer des Hauses übernommen hat. Zudem wird dort auch eine weitere Gebühr von 20.000 USD aufgeführt, die von den „Managing Members“ übernommen wird. Aus der Tabelle „Use of proceeds to issuer“ ist überdies zu sehen, dass es keine „Offering Expenses“ gegeben hat, weil die Angebotskosten (Prospektkosten etc.) auch durch die „Managing Members“ übernommen wurden. Als einziger Kostenblock in der Tabelle „Use of proceeds to issuer“ wird der Betrag von 6450 USD für „Working Capital" aufgeführt.

Ein interessanter Aspekt des 9943 Marlove-Beispiel ist, dass die Herausgeber des Tokens, viele Kosten nicht verrechnet haben. Es lässt sich vermuten, dass sie in der Testphase beim ersten Objekt gewisse Kosten selber zu übernehmen bereit waren, um die Token für die Investoren attraktiv zu gestalten und sich langfristig

\footnotetext{
56 Das offizielle Dokument zu diesem Projekt befindet sich auf https://realt.co/wp-content/uploads/2019/ 09/REALTOKEN-LLC-SERIES-1-9943-MARLOWE-1.pdf. Gesehen am 19. Februar 2020.

57 Informationen zum Objekt befinden sich auf https://realt.co/product/9943-marlowe-st-detroit-mi48227/. Diese Seite wurde aufgerufen am 17. Februar 2020.

58 Die Wertsteigerung des Tokens lässt sich auf zwei mögliche Gründe zurückführen. Entweder hat sich der innere Wert der Immobilie vom äußeren Wert des Tokens entkoppelt oder die Immobilie ist im Wert gestiegen. Informationen von Uniswap wurde am 19.02.2020 abgerufen auf: https://uniswap.info/token/ 0xe5f7ef61443fc36ae040650aa585b0395aef77c8.

59 Auf unsere Anfrage zu Emissionskosten beantwortete ein Mitarbeiter von RealT, dass sie bei der Emission eine Pauschale pro Token von zehn Prozent erheben. Um ihre laufenden Kosten zu decken, wird 2,5\% der Bruttomiete als eine weitere Gebühr erhoben.

60 Vgl. RealT (2019a). Entnommen dem offiziellen Dokument: PRIVATE PLACEMENT MEMORANDUM AMENDED AS OF AUGUST 15, 2019 S. 35
} 
We estimate that the gross proceeds of this Offering will be approximately $\$ 63,750.00$ assuming the full amount of this Offering is sold, and will be used as follows:

\begin{tabular}{|c|c|c|}
\hline Uses & $\begin{array}{c}\text { Dollar } \\
\text { Amount }\end{array}$ & $\begin{array}{c}\text { Percentage of } \\
\text { Gross Cash } \\
\text { Proceeds } \\
\end{array}$ \\
\hline Purchase Price of the Underlying Asset ${ }^{(1)}$ & $\$ 57,300.00$ & $89.88 \%$ \\
\hline Investment Banking Advisor Fees ${ }^{(2)}$ & $\$ 0.00$ & $0.00 \%$ \\
\hline Offering Expenses $^{(3)}$ & $\$ 0.00$ & $0.00 \%$ \\
\hline Acquisition Expenses $^{(4)}$ & $\$ 0.00$ & $0.00 \%$ \\
\hline Working Capital() & $\$ 6,450.00$ & $10.12 \%$ \\
\hline Total Fees and Expenses & $\$ 63,750.00$ & $100.00 \%$ \\
\hline Totsl Proceeds & $\$ 63,750.00$ & $100.00 \%$ \\
\hline
\end{tabular}

(1) Consists of the Note in the principal amount of $\$ 57,300$ issued by RealT to the Asset Seller.

(2) The $5 \%$ Cash Service Fee to the Investment Banking Advisor, which will total, $\$ 3,187.50$, will be borne by the Asset Seller. Does not include an estimated $\$ 20,000$ in expense reimbursements to be paid to the Investment Banking Advisor by the Managing Member. The Investment Banking Advisor Cash Service Fee will not be paid on the gross proceeds from the sale of any Interests purchased by the Managing Member or its affiliates.

(3) Solely in connection with this Offering, the Managing Member has assumed, and will not be reimbursed for, the Offering Expenses.

(4) Although Acquisition Expenses are typically built into the Purchase Price of the Underlying Asset, the Series may incur certain post-Closing acquisition related expenses such as property appraisal costs.

(5) Includes $3 \%$ per annum interest accrued on the Note to Asset Seller.

Abb. 1 Use of proceeds to issuer

als Technologie-Vorreiter auf dem Markt zu etablieren. In der Zwischenzeit, wie in der Tab. 1 dargestellt wird, hat die RealToken $\mathrm{GmbH}$ viele weiter Immobilien tokenisiert. $^{61}$

Die Tokenisierung von Immobilien steckt in der absoluten Pionierphase. Die Tokenisierung der 9943 Marlove-Immobilie ist nach unserem Wissen weltweit die erste Tokenisierung einer Immobilie, bei der anschliessend der Token auch weltweit rund um die Uhr handelbar gemacht wurde. In dieser Anfangsphase ist es deshalb schwierig, einen Kostenvergleich mit traditionellen Immobilienanlageprodukten vorzunehmen. Die Emissionskosten von offenen oder geschlossenen Fondsanteilen betragen zwischen fünf und fünfzehn Prozent (die sogenannten weichen Kosten). Damit dürfte für einen Emittenten eine Kapitalbeschaffung über eine Tokenisierung zurzeit teurer sein als eine Finanzierung mit herkömmlichen Finanzierungsinstrumenten.

Weil die RealToken GmbH laufend neue Immobilien tokenisiert, müssen die Token der einzelnen Immobilien unterscheidbar sein. Jede Immobilie hat ihr eigenes Rechtskonstrukt (genannt Serie) und der dazugehörige Token eine eindeutige Identifikationsnummer (UIN). ${ }^{62}$ Das bedeutet, dass die Token von zwei unterschiedlichen Immobilien nicht fungibel sind. Alle RealToken mit der gleichen Identifikationsnummer sind aber fungibel.

Das Halten von Token einer bestimmten Serie von RealToken GmbH verleiht dem Besitzer einen proportionalen Anteil am Cashflow der der Serie zugewiesenen Immobilie. Aus der Urkunde einer Serie geht hervor, welche Immobilie sie umfasst,

61 Eigen Darstellung in Anlehnung an https://realt.co/marketplace/ (Stand 20. Juli 2020).

${ }^{62}$ Eine Serie ist vergleichbar mit einer Zweckgesellschaft (SPV) in der traditionellen Verbriefung. 
Tab. 1 Tokenisierte Immobilien

\begin{tabular}{lllll}
\hline $\begin{array}{l}\text { Immobilie/Token- } \\
\text { Name }\end{array}$ & $\begin{array}{l}\text { Token-Preis } \\
\text { (USD) }\end{array}$ & $\begin{array}{l}\text { Token-Ge- } \\
\text { samtanzahl }\end{array}$ & $\begin{array}{l}\text { Mieteinnahmen } \\
\text { pro Token (\%) }\end{array}$ & $\begin{array}{l}\text { Mieteinnahmen/ } \\
\text { Token/Jahr (USD) }\end{array}$ \\
\hline 9943 Marlowe & 65,75 & 1000 & 12,34 & 7,90 \\
16200 Fullerton & 161,84 & 3800 & 12,76 & 20,65 \\
9336 Paten & 62,70 & 1000 & 10,25 & 6,42 \\
20200 Lesure & 69,40 & 1000 & 10,25 & 7,11 \\
5942 Audubon & 77,73 & 750 & 10,38 & 8,07 \\
10024-28 Appoline & 145,56 & 4000 & 11,82 & 16,70 \\
8342 Schaefer & 50,83 & 3000 & 12,59 & 6,40 \\
25097 Andover & 53,13 & 1400 & 11,06 & 5,88 \\
18276 Appoline & 52,32 & 1400 & 11,90 & 6,23 \\
18900 Mansfield & 51,31 & 1100 & 11,09 & 5,69 \\
15634 Liberal & 48,98 & 1200 & 13,04 & 6,39 \\
15048 Freeland & 49,02 & 1300 & 11,66 & 5,72 \\
272 42nd Court & 57,96 & 3000 & 7,00 & 4,06 \\
9165 Kensington & 52,91 & 1300 & 11,63 & 6,15 \\
10084 Grayton & 50,73 & 1300 & 11,27 & 5,67 \\
\hline
\end{tabular}

sowie alle wichtigen Informationen zum Erwerb dieser Serie. ${ }^{63}$ Diese Urkunde wird in dem Bezirk hinterlegt, in dem sich die Liegenschaft befindet, und anschließend öffentlich registriert. ${ }^{64}$

Die Besitzer der Token erhalten tägliche Auszahlungen, die aus den Mieteinnahmen generiert werden. Dies wird dadurch erreicht, dass der Smart Contract jeden Tag den öffentlichen Adressen, die ein Token-Guthaben haben, eine Mikrozahlung gutschreibt. Dabei handelt es sich um eine Verrechnung, die innerhalb des Smart Contracts stattfindet. Der Emittent muss zu diesem Zweck sicherstellen, dass die Auszahlungsadresse ein hinreichend großes Guthaben an der DAI-Kryptowährung besitzt. ${ }^{65}$

Gemäß der Securities and Exchange Commission (SEC) ist der Handel mit den RealToken in den Vereinigten Staaten nach dem Wertpapiergesetz 1933 in der derzeit gültigen Fassung nicht zugelassen. ${ }^{66}$ Dadurch dürfen die RealToken in den Vereinig-

\footnotetext{
63 Alle Token einer Serie besitzen jeweils die gleiche UIN, die sowohl im Token als auch im Gründungszertifikat der RealToken GmbH hinterlegt ist. Das Gründungszertifikat wird laufend erweitert durch die Zufügung von neuen Serien. Diese Informationen werden auch auf der Ethereum-Blockchain abgesichert.

64 Mit der Einreichung der Urkunde muss gleichzeitig eine eidesstattliche Erklärung vorliegen, aus der hervorgeht, dass die in der Urkunde aufgeführte und beschriebene Serie mit dem tatsächlichen Eigentum übereinstimmt und eine eindeutige Identifikationsnummer aufweist.

65 Der Marktpreis eines Wertpapieres, das eine Dividende auszahlt, fällt in der Regel nach der Auszahlung der Dividende. Diese Preisschwankungen werden kleiner, je öfter eine Dividende ausbezahlt wird. Der Vorteil einer täglichen Dividende ist, dass der Wert des Wertpapiers vor und nach der Auszahlung einer Dividende nur wenig schwankt.

66 SEC Informationen befinden sich auf https://www.sec.gov/files/dlt-framework.pdf. Gesehen am 02. Juli 2020 .
} 
ten Staaten nur an akkreditierte Anleger verkauft werden. ${ }^{67}$ Das Dokument „Private Placement Memorandum Amended As Of August, 2019“ der RealToken GmbH enthält eine Liste im Anhang A, welche die Verkaufsbeschränkungen für etwa zwanzig weitere Länder aufzählt.

Beim Kauf eines RealToken über die RealToken-Plattform oder über einen Sekundärmarkt wird jeder Anleger dahingehend überprüft, ob die Anforderungen an die Akkreditierung (falls vorhanden) des Investors erfüllt sind. Zusätzlich muss sich jeder Anleger identifizieren (KYC) und das AML-Verfahren zur Bekämpfung der Geldwäsche unterzeichnen. Das KYC-Verfahren dient zur Überprüfung der Identität der RealToken-Käufer, während das AML-Verfahren sicherstellen soll, dass Zahlungen für RealToken nicht aus illegalen Quellen stammen.

Zum obigen Beispiel sollte auch der BrickMark Token erwähnt werden. ${ }^{68}$ Die BrickMark AG kaufte am 15. Januar 2020 eine Immobilie an der Bahnhofstrasse 52, Zürich, für 130 Mio. CHF. Dabei wurde ein erheblicher Teil des Kaufpreises mit BrickMark-Token bezahlt. ${ }^{69}$ Der BrickMark-Token wurde auf einer permissioned Blockchain ausgegeben und ist nicht öffentlich handelbar. ${ }^{70}$

\section{Zusammenfassung}

Der vorliegende Artikel gibt Aufschluss über die Technologie und die Regulierung, die bei der Tokenisierung einer Immobilie und dem anschließenden Handel des Tokens zur Anwendung kommt. Für den Eigentümer einer Immobilie ist der Vorteil der Tokenisierung, dass sie ihm ermöglicht, durch die Ausgabe von Token schnell und unkompliziert liquide Mittel zu beschaffen. Nach der Emission sind die Token unmittelbar weltweit auf der öffentlich zugänglichen Ethereum-Plattform handelbar und zwar rund um die Uhr. Auf dieser Plattform ist ein Eigentumsübertrag von Token schon in wenigen Sekunden abgeschlossen. Ein Token-Käufer braucht für den Erwerb nur ein ERC20 kompatibles Wallet. Die weiteren Vorteile der Tokenisierung, auf die in diesem Artikel eingegangen wurde, sind Standardisierung, Flexibilität, die

\footnotetext{
67 Darunter versteht man Investoren mit einem besonderen Status. Die Definition eines akkreditierten Investors (falls vorhanden) und die Folgen einer Einstufung als solcher sind von Land zu Land unterschiedlich. Die Definition eines akkreditierten Anlegers in den Vereinigten Staaten ist abrufbar auf https:// www.ecfr.gov/cgi-bin/retrieveECFR?gp=\&SID=8edfd12967d69c024485029d968ee737\&r=SECTION\& $\mathrm{n}=17 \mathrm{y} 3.0 .1 .1 .12 .0 .46 .176$. Gesehen am 02. Juli 2020.

68 Vgl. Brickmark auf https://brickmark.io/. Gesehen am 10. März 2020.

69 Viele Informationen dieser Zusammenfassung gehen auf die Telefonkonferenz zurück, die am 15. Januar 2020 stattgefunden hat und am 20. Februar 2020 veröffentlicht wurde auf https://webcasts.eqs.com/ brickmark20200115/no-audio. Gesehen am 10. März 2020.

70 Obwohl der Token momentan weder direkt noch auf den sekundären Märkten handelbar ist, akzeptierte der Käufer den Token und betonte: „We gladly accepted the Brickmark tokens as part of the purchase price. We assume that digital financial instruments will also significantly gain in importance in the real estate sector in the future. The current transaction may have an icebreaker function for the sector in terms of its volume and institutional character." Siehe auf https://medium.com/brickmark-ag/brickmark-signspurchase-agreement-for-the-largest-ever-real-estate-transaction-paid-in-tokens-of-13a6195cb303. Gesehen am 10. März 2020.
} 
hohe Sicherheit, Transparenz und, dass eine beliebig kleine Stückelung eines Tokens möglich ist.

Im Anschluss an die theoretische Analyse wurde ein konkretes Tokenisierungsprojekt präsentiert, nämlich die Tokenisierung einer Immobilie unter dem TokenNamen 9943 Marlowe. Dabei stellte sich heraus, dass einige der theoretischen Vorteile in der Praxis bislang nicht zur Geltung kommen. Der Grund hierfür sind die bestehenden Unsicherheiten bezüglich der finanzmarktrechtlichen Regulierung von Token. Viele Länder haben noch keine umfassenden Rechtsvorschriften ausgearbeitet, auf die man sich als Token-Emittent oder Investor berufen kann. Dies führt zurzeit dazu, dass viele Token-Emittenten die Handelbarkeit zum aktuellen Zeitpunkt noch einschränken.

Neben den regulatorischen Unsicherheiten gibt es auch weitere Herausforderungen. Die Ethereum-Plattform steht zurzeit unter einer hohen Auslastung, was zu hohen Transaktionsgebühren führt. Ferner wird auch der Energieverbrauch des proof-of-work Konsensus-Mechanismus, der bei Ethereum zurzeit zur Anwendung kommt, stark kritisiert. Die neue Software Ethereum 2.0 soll mittelfristig beide Probleme lösen.

Das Internet hat gezeigt, dass neue Modelle und Verhaltensweisen entstehen, wenn die Kosten der Kommunikation vernachlässigbar klein werden. Seine Geschichte hat uns auch gelehrt, dass die Auswirkungen einer solchen Entwicklung auf die Gesellschaft und Wirtschaft nicht vorhersehbar sind. Die Blockchain-Technologie erlaubt theoretisch den weltweiten Austausch von Werten zu vernachlässigbar kleinen Kosten. Die Tokenisierung ermöglicht den weltweiten Handel rund um die Uhr von illiquiden Vermögenswerten. Ob sich diese Technologien etablieren werden und wie sich diese auf Gesellschaft und Wirtschaft auswirken werden, bleibt offen.

\footnotetext{
Danksagung Wir bedanken uns bei zwei anonymen Gutachtern und Editoren für wertvolle Kommentare und Anregungen. Ebenso möchten wir uns bei Herrn Prof. Wolfgang Buchholz und Herrn Prof. Wolfgang Schäfers für ihre konstruktive Kritik sowie bei der Federal Reserve Bank of St. Louis für die Unterstützung bedanken. Alle verbleibenden Fehler sind unsere eigenen.
}

Open Access Dieser Artikel wird unter der Creative Commons Namensnennung 4.0 International Lizenz veröffentlicht, welche die Nutzung, Vervielfältigung, Bearbeitung, Verbreitung und Wiedergabe in jeglichem Medium und Format erlaubt, sofern Sie den/die ursprünglichen Autor(en) und die Quelle ordnungsgemäß nennen, einen Link zur Creative Commons Lizenz beifügen und angeben, ob Änderungen vorgenommen wurden.

Die in diesem Artikel enthaltenen Bilder und sonstiges Drittmaterial unterliegen ebenfalls der genannten Creative Commons Lizenz, sofern sich aus der Abbildungslegende nichts anderes ergibt. Sofern das betreffende Material nicht unter der genannten Creative Commons Lizenz steht und die betreffende Handlung nicht nach gesetzlichen Vorschriften erlaubt ist, ist für die oben aufgeführten Weiterverwendungen des Materials die Einwilligung des jeweiligen Rechteinhabers einzuholen.

Weitere Details zur Lizenz entnehmen Sie bitte der Lizenzinformation auf http://creativecommons.org/ licenses/by/4.0/deed.de.

\section{Literatur}

Ang A (2014) Asset management-a systematic approach to factor investing. Oxford University Press, Oxford 
Anson M, Fabozzi F, Jones F (2011) The handbook of traditionaland alternative investment vehicles: investment characteristics and strategies. Wiley, Hoboken

Antonopoulos AM (2017) Mastering Bitcoin: programming the open Blockchain, 2. Aufl. O’Reilly Media, Newton

Antonopoulos AM, Wood G (2018) Mastering Ethereum: building smart contracts and DApps. O’Reilly Media, Inc, USA

BaFin (2018) Digitalisierung: Folgen für Finanzmarkt, Aufsicht und Regulierung - Teil I. https:// www.bafin.de/SharedDocs/Downloads/DE/BaFinPerspektiven/2018/bp_18-1_digitalisierung.pdf? blob=publicationFile \&v=18. Zugegriffen: 3. Juli 2020

BaFin (2019a) Krypto-Token, Merkblatt der BaFin zu Prospekt- und Erlaubnispflichten. Zweites Hinweisschreiben zu Prospekt- und Erlaubnispflichten im Zusammenhang mit der Ausgabe sogenannter Krypto-Token. https://paytechlaw.com/krypto-token-bafin/. Zugegriffen: 3. Juli 2020

BaFin (2019b) Tokenisierung. https://www.bafin.de/dok/12342268. Zugegriffen: 3. Juli 2020

Berentsen A, Schär F (2017) Bitcoin, Blockchain und Kryptoassets: Eine umfassende Einführung. Books on Demand, Nordersted

Berentsen A, Schär F (2019) Stablecoin: the quest for a low-volatility cryptocurrency. In: Fatas A (Hrsg) The economics of Fintech and digital currencies. CEPR Press, London, UK. https://voxeu.org/ content/economics-fintech-and-digital-currencies. Zugegriffen: 27. März 2020

Berentsen A, Schär F (2020) Bitcoin, Blockchain, and Cryptoassets: a comprehensive introduction. MIT Press, Cambridge, Massachusetts London, England

Buterin V (2013) A next-generation Smart Contract and decentralized application platform Ethereum. White Paper. https://github.com/ethereum/wiki/wiki/White-Paper. Zugegriffen: 27. März 2020

Buterin V, Vogelstellert F (2015) ERC-20 Token Standard. https://eips.ethereum.org/EIPS/eip-20. Zugegriffen: 27. März 2020

Cheng P, Zhenguo L, Yingchun L (2013) Liquidity risk of private assets: evidence from real estate markets. Financial Rev 48(4):671-696

Damodaran A (2005) Marketability and value: measuring the Illiquidity discount. https://ssrn.com/ abstract=841484. https://doi.org/10.2139/ssrn.841484. Zugegriffen: 9. September 2020

dejure (2020) Wertpapierhandelsgesetz (WpHG). https://dejure.org/gesetze/WpHG. Zugegriffen: 27. März 2020

Diffie W, Hellman ME (1976) New directions in cryptography. IEEE Transactions on Information Theory 22(6):644-654. https://ieeexplore.ieee.org/document/1055638. Zugegriffen 8. Januar 2021

EU (2014a) Direktive. http://data.europa.eu/eli/dir/2014/65/oj. Zugegriffen: 2. Juli 2020

EU (2014b) Richtlinie 2014/65/EU. https://www.finanzmarktregulierung-umsetzen.de/wp-content/uploads/ 2014/06/20140620_Richtlinine_MiFID-II_Amtsblatt-EU.pdf. Zugegriffen: 27. März 2020

FINMA (2018) ICOs guidelines. https://www.finma.ch/de/news/2018/02/20180216-mm-ico-wegleitung/. Zugegriffen: 27. März 2020

Fußwinkel O, Kreiterling C (2018) Blockchain-Technologie - Gedanken zur Regulierung. https:// www.bafin.de/SharedDocs/Veroeffentlichungen/DE/BaFinPerspektiven/2018/bp_18-1_Beitrag_ Fusswinkel.html. Zugegriffen: 20. Sept. 2020

Haber S, Stornetta WS (1991) How to time-stamp a digital document. J Cryptol 3:99-111. https://doi.org/ $10.1007 / \mathrm{BF} 00196791$

Jobst A (2008) What is securitization? Finance Dev 45(3):48-49. https://www.imf.org/external/pubs/ft/ fandd/2008/09/pdf/fd0908.pdf. Zugegriffen: 27. März 2020

Nakamoto S (2008) Bitcoin: a peer-to-peer electronic cash system. The Bitcoin whitepaper. https://www. bitcoin.com/bitcoin.pdf. Zugegriffen: 27. März 2020

Narayanan A, Bonneau J, Felten E, Miller A, Goldfeder S (2016) Bitcoin and cryptocurrency technologies: a comprehensive introduction. Princeton University Press, Princeton

Nash J (1951) Non-cooperative games. Ann Math Second Ser 54(2):286-295. https://doi.org/10.2307/ 1969529

Nyffenegger R, Schär F (2018) Token Sales: Eine Analyse des Blockchain-basierten Unternehmensfinanzierungsinstruments. Corporate Finance:21-125. https://edoc.unibas.ch/68783/. Zugegriffen: 27. März 2020

ProspVO (2017) Verordnung (EU) 2017/1129 des Europäischen Parlaments und des Rates vom 14. Juni 2017 über den Prospekt, der beim öffentlichen Angebot von Wertpapieren oder bei deren Zulassung zum Handel an einem geregelten Markt zu veröffentlichen ist und zur Aufhebung der Richtlinie 2003/71/EGText von Bedeutung für den EWR. ELI: http://data.europa.eu/eli/reg/2017/1129/201912-31. Zugegriffen: 8. Januar 2021 
ProspVO (2019) Verordnung (EU) 2017/1129 des Europäischen Parlaments und des Rates vom 14. Juni 2017 über den Prospekt, der beim öffentlichen Angebot von Wertpapieren oder bei deren Zulassung zum Handel an einem geregelten Markt zu veröffentlichen ist und zur Aufhebung der Richtlinie 2003/71/EG (Text von Bedeutung für den EWR)Text von Bedeutung für den EWR. ELI: http://data. europa.eu/eli/reg/2017/1129/2019-12-31. Zugegriffen: 8. Januar 2021

RealT (2019a) Private placement memorandum amended AS of August. https://realt.co/wp-content/ uploads/2019/09/REALTOKEN-LLC-SERIES-1-9943-MARLOWE-1.pdf. Zugegriffen: 27. März 2020

RealT (2019b) Whitepaper. https://realt.co/wp-content/uploads/2019/05/RealToken_White_Paper_US_ v03.pdf. Zugegriffen: 27. März 2020

Savills Research (2016) What Price the World? Trends in international real estate trading. https://pdf. euro.savills.co.uk/global-research/around-the-world-in-dollars-and-cents-2016.pdf. Zugegriffen: 23. März 2020

Szabo N (1996) Smart contracts: building blocks for digital markets. http://www.fon.hum.uva.nl/ rob/Courses/InformationInSpeech/CDROM/Literature/LOTwinterschool2006/szabo.best.vwh.net/ smart_contracts_2.html. Zugegriffen: 10. Juli 2020

Voshmgir S (2019a) Token economy: how blockchains and smart contracts revolutionize the economy, S 87-98

Voshmgir S (2019b) contribution. In: Rechtshandbuch Smart Contracts. C.H.Beck, Verlag

de Vries A (2018) Bitcoin's growing energy problem. Joule 2:801-805. https://doi.org/10.1016/j.joule. 2018.04.016

Wälti S (2020) DLT-Gesetz: Nationalrat stimmt Anpassungen des Bundesrechts zu. https://www. wickipartners.ch/news/dlt-gesetz-nationalrat-anpassungen-des-bundesrechts. Zugegriffen: 30 . Juli 2020

WEF (2015) lobal Agenda Council on the Future of Software \& SocietyDeep ShiftTechnology Tipping Points and Societal ImpactSurvey Report. http://www3.weforum.org/docs/WEF_GAC15_ Technological_Tipping_Points_report_2015.pdf. Zugegriffen: 27. März 2020 